\title{
TITLE:
}

\section{What happens when the inflaton stops during inflation}

$\operatorname{AUTHOR}(S)$ :

Seto, O; Yokoyama, J; Kodama, H

CITATION:

Seto, $O$...[et al]. What happens when the inflaton stops during inflation. PHYSICAL REVIEW D 2000, 61(10): 103504.

\section{ISSUE DATE:}

2000-05-15

URL:

http://hdl.handle.net/2433/50474

RIGHT:

Copyright 2000 American Physical Society 
PHYSICAL REVIEW D, VOLUME 61, 103504

\title{
What happens when the inflaton stops during inflation
}

\author{
Osamu Seto \\ Yukawa Institute for Theoretical Physics, Kyoto University, Kyoto 606-8502, Japan
}

Jun'ichi Yokoyama

Isaac Newton Institute for Mathematical Sciences, University of Cambridge, Cambridge, CB3 OEH, United Kingdom and Department of Earth and Space Science, Graduate School of Science, Osaka University, Toyonaka 560-0043, Japan

\author{
Hideo Kodama \\ Yukawa Institute for Theoretical Physics, Kyoto University, Kyoto 606-8502, Japan \\ (Received 8 November 1999; published 17 April 2000)
}

\begin{abstract}
The spectrum of adiabatic density perturbation generated during inflation is studied in the case the time derivative of an inflation-driving scalar field (inflaton) vanishes at some time during inflation. It is shown that the nondecaying mode of perturbation has a finite value even in this case and that its amplitude is given by the standard formula with the time derivation of the scalar field replaced by the potential gradient using the slow-roll equation.

PACS number(s): $98.80 . \mathrm{Cq}$
\end{abstract}

\section{INTRODUCTION}

It is now widely believed that the large-scale homogeneity and isotropy observed in the Universe were realized as a result of accelerated expansion or inflation in the early universe [1]. It also provides a mechanism to account for the origin of primordial density fluctuations out of quantum fluctuations of the inflation-driving scalar field which we call the inflaton [2]. In the standard inflation models such as new [3] and chaotic [4] inflation, inflation is driven by the potential energy of the inflation as it slowly rolls the potential hill and is predicted to produce adiabatic fluctuations with a nearly scale-invariant spectrum.

More specifically, the amplitude of curvature perturbation, $\Phi$, on a comoving scale $r=2 \pi / k$ is given by the formula

$$
\left.\Phi(r) \approx \frac{H^{2}}{|\dot{\phi}|}\right|_{t_{k}},
$$

where $\phi$ is the inflaton and $H$ is the Hubble parameter during inflation, and the right-hand side should be evaluated when the relevant scale left the Hubble radius during inflation. The above formula also gives an estimate of the amplitude of density perturbation on the comoving scale $r$ when this scale reentered the Hubble horizon after inflation as well as that of large-scale anisotropy of cosmic microwave background due to the Sachs-Wolfe effect [5], which has been probed by the Cosmic Background Explorer satellite [6]. The reason why Eq. (1) gives an almost scale-invariant spectrum is that both $H$ and $\dot{\phi}$ change very slowly during slow-roll inflation.

Recently, however, new classes of inflation models have been proposed such that $\phi$ is not necessarily slowly rolling during the entire period of inflation and that it changes direction of motion during inflation. One example is the oscillating inflation proposed by Damour and Mukhanov $[7,8]$ in which accelerated expansion is realized as the inflaton oscil- lates around a minimum of a nonconvex potential. In this model $\dot{\phi}$ vanishes twice in each period of oscillation during inflation. Another example is the chaotic new inflation model proposed by one of us [9]. This model assumes a potential with a local maximum at the origin like new inflation but start with the same initial condition as chaotic inflation. If model parameters are appropriately chosen, the inflaton climbs up the potential hill near to the origin after chaotic inflation and new inflation can be realized there. In the early stage of new inflation $\dot{\phi}$ may vanish and $\phi$ changes its direction of motion if it does not have sufficient energy to go over the origin.

In both models, if we apply the formula (1) as it is, the amplitude of fluctuation apparently diverges when $\dot{\phi}$ vanishes. The above formula, however, has been derived under the slow-roll approximation, namely, under the assumption that both $\phi$ and $\Phi$ changes slowly during inflation. On the other hand, motion of $\phi$ is not given by the slow-roll formula when $\phi$ changes its direction, because $\dot{\phi}$ vanishes where the gradient of the potential does not vanish.

Thus we expect that it is inappropriate to apply Eq. (1) to the case $\dot{\phi}$ vanishes during inflation. The purpose of the present paper is to derive a formula of curvature perturbation in such a situation. This is accomplished by a proper account of not only the growing (or nondecreasing) mode but also the decaying mode as seen below. The rest of the paper is organized as follows. In Sec. II we give the formulation with an appropriate choice of a variable and present solutions in the long- and the short-wavelength limit. Then these solutions are matched for the case of the linear potential in Sec. III and for the quadratic potential in Sec. IV. Section V is devoted to the conclusion.

\section{EQUATIONS OF MOTION AND THE SOLUTIONS IN THE LONG- AND THE SHORT-WAVELENGTH LIMIT}

We consider a minimally coupled singlet scalar field $\phi$ in the spatially flat Robertson-Walker background metric: 


$$
d s^{2}=-d t^{2}+a^{2}(t) d \mathbf{x}^{2}
$$

where $a(t)$ denotes the scale factor. Then the total action is

$$
S=\frac{1}{2 \kappa^{2}} \int R \sqrt{-g} d^{4} x+\int\left[-\frac{1}{2}(\partial \phi)^{2}-V(\phi)\right] \sqrt{-g} d^{4} x,
$$

where $R$ is the scalar curvature and $\kappa^{2}=8 \pi G$ with $G$ being the gravitational constant. The Einstein equation and the field equation of $\phi$ read

$$
\begin{gathered}
H^{2}=\left(\frac{\dot{a}}{a}\right)^{2}=\frac{\kappa^{2}}{3}\left(\frac{1}{2} \dot{\phi}^{2}+V(\phi)\right), \\
\ddot{\phi}+3 H \dot{\phi}+V^{\prime}(\phi)=0,
\end{gathered}
$$

with $H$ being the Hubble parameter. Here a dot denotes time differentiation and a prime represents differentiation with respect to $\phi$. These are the equations for the unperturbed variables.

Next we incorporate a linear perturbation, writing the perturbed metric in terms of the gauge-invariant variables [10] in the longitudinal gauge,

$$
d s^{2}=-[1+2 \Psi(\mathbf{x}, t)] d t^{2}+a^{2}(t)[1+2 \Phi(\mathbf{x}, t)] d \mathbf{x}^{2},
$$

where we use the notation of [11] for the perturbation variables. Hereafter all perturbation variables represent Fourier expansion coefficients such as

$$
\Phi_{\mathbf{k}}=\int \frac{d^{3} \mathbf{x}}{(2 \pi)^{3 / 2}} \Phi(\mathbf{x}, t) e^{i \mathbf{k} \cdot \mathbf{x}}
$$

and we omit the wave-number suffix $\mathbf{k}$. We use the following combination of gauge-invariant variables [12-14]:

$$
Y=X-\frac{\dot{\phi}}{H} \Phi
$$

where

$$
X=\delta \phi-\frac{a}{k} \dot{\phi} \sigma_{g}
$$

is the gauge-invariant scalar field fluctuation with $\sigma_{g}$ being the shear of each constant time slice. The latter vanishes on Newtonian slice including the longitudinal gauge.

The above quantity $Y$ is related to the gauge-invariant variable

$$
Z \equiv \Phi-\frac{a H}{k} V=\Phi+\frac{2}{3} \frac{\Phi+H^{-1} \Phi}{1+w},
$$

as

$$
Z=-\frac{H}{\dot{\phi}} Y
$$

in the present case where matter consists of a scalar field. Here $V$ is a gauge-invariant velocity perturbation [11] and $w$ denotes the ratio of pressure to energy density. Although the definition of $Z$ is slightly different from the Bardeen's $\zeta$ [15], which was originally defined by

$$
\zeta=\left[1+\frac{2}{9} \frac{k^{2}}{(1+w) H^{2} a^{2}}\right] \Phi-\frac{a H}{k} V
$$

it enjoys the same property as $\zeta$, that is, both quantities are constant during $k \ll a H$ if only adiabatic fluctuation is present and sound velocity is nonsingular.

From the perturbed Einstein equations, we obtain the following equations:

$$
\begin{gathered}
\dot{X} \dot{\phi}-\ddot{\phi} X+\dot{\phi}^{2} \Phi=\frac{2}{\kappa^{2}} \frac{k^{2}}{a^{2}} \Phi, \\
\Phi+H \Phi=-\frac{\kappa^{2}}{2} \dot{\phi} X .
\end{gathered}
$$

From these equations and Eq. (8), the equation of motion of $Y$ reads

$$
\ddot{Y}+3 H \dot{Y}+\left[\left(\frac{k}{a}\right)^{2}+M_{Y \text { eff }}^{2}\right] Y=0
$$

with

$$
M_{Y \text { eff }}^{2} \equiv V^{\prime \prime}(\phi)+3 \kappa^{2} \dot{\phi}^{2}-\frac{\kappa^{4}}{2 H^{2}} \dot{\phi}^{4}+2 \kappa^{2} \frac{\dot{\phi}}{H} V^{\prime}(\phi) .
$$

This equation has the following exact solution in the longwavelength limit $k \rightarrow 0[13,16]$ :

$$
\begin{gathered}
Y(t)=\widetilde{c}_{1}(k) Y_{1}(t)+\widetilde{c}_{2}(k) Y_{2}(t), \\
Y_{1}(t)=\frac{\dot{\phi}}{H}
\end{gathered}
$$

$$
Y_{2}(t)=\frac{\dot{\phi}}{H} \int_{T}^{t} \frac{H^{2}}{a^{3} \dot{\phi}^{2}} d t
$$

where $\widetilde{c}_{1}(k)$ and $\widetilde{c}_{2}(k)$ are integration constants to be determined by quantum fluctuations generated during inflation, and $T$ is some initial time which may be chosen arbitrarily because its effect can be absorbed by a redefinition of $\widetilde{c}_{1}(k)$. The solution $Y_{2}(t)$ is apparently singular at $\dot{\phi}=0$. But, in fact, it is regular there [13].

Since $Y_{2}(t)$ has a different dimension than $Y_{1}(t)$, it is more convenient to redefine the coefficients as

$$
c_{1}(k) \equiv \widetilde{c}_{1}(k), \quad c_{2}(k) \equiv \widetilde{c}_{2}(k) / k^{3},
$$

for which the scale factor appears in the rescaling-invariant form of $k / a(t)$, and $c_{1}(k)$ and $c_{2}(k)$ have the same dimension. 
We next consider the evolution of $Y$ in the shortwavelength regime in order to set the initial condition of $Y$ out of quantum fluctuations. Since we are interested in the generation of perturbations around the turning point of the inflaton, we only consider the evolution of $Y$ in the inflationary stage around and after the time $\dot{\phi}=0$ and assume that $\left|\dot{H} / H^{2}\right| \ll 1$ holds in this stage. We further assume that $\mu$ $\equiv V^{\prime} /(\kappa V)$ satisfies the condition

$$
\mu^{\prime} \leqslant 0, \quad \mu \mu^{\prime \prime} \geqslant 0
$$

for the value of $\phi$ in this stage. This condition is satisfied for quite a large class of potentials including the pure exponential potential and those which are approximately given by $c|\phi|^{n}$ or $V_{0}-c|\phi|^{n}$ around the turning point where $c>0$ and $n$ is a positive integer.

$M_{Y \text { eff }}^{2}$ defined by Eq. (16) can be rewritten in terms of $\mu$ as

$$
\frac{M_{Y \text { eff }}^{2}}{\kappa^{2} V}=\frac{1}{\kappa} \mu^{\prime}+\left(\frac{\kappa \dot{\phi}}{H}+\mu\right)^{2}
$$

As is shown in the Appendix, under condition (21), the value of $\left|\mu^{\prime}\right| / \kappa$ at the turning point should be smaller than $1 / N$, where $N$ is the number of $e$-folds of inflation after the turning point, i.e., the increase of $\ln a$. There it is also shown that $|\kappa \dot{\phi} /(H \mu)|$ is always less than unity and approaches unity, i.e., $\kappa \dot{\phi} /(H \mu) \rightarrow-1$, in an expansion time after $\phi$ passes the turning point. This implies that the second term in the righthand side of Eq. (22) is equal or less than the value of $2\left|\dot{H} / H^{2}\right|$ at the slow-roll phase following the short transient period around the turning point. To be precise, this term is approximately given by $(2 / 9)\left(\left|\mu^{\prime}\right| / \kappa\right)^{2}\left|\dot{H} / H^{2}\right|$ in the slowroll phase.

From these estimates we see that $M_{Y \text { eff }}^{2}$ in Eq. (15) can be neglected in the regime

$$
\left(\frac{k}{a H}\right)^{2}>\frac{1}{N}
$$

which extends to a wavelength much larger than the Hubble horizon size. Thus in this regime Eq. (15) becomes

$$
\ddot{Y}+3 H \dot{Y}+\left(\frac{k}{a}\right)^{2} Y=0 .
$$

Under the condition $\left|\dot{H} / H^{2}\right| \ll 1$ the solution of this equation satisfying the normalization condition for positive frequency modes to define the vacuum state,

$$
a^{3}\left(Y \dot{Y} *-\dot{Y} Y^{*}\right)=i,
$$

is approximately given by

$$
Y=\frac{i H}{\sqrt{2 k^{3}}}\left[\alpha_{\mathbf{k}}(1+i k \eta) e^{-i k \eta}-\beta_{\mathbf{k}}(1-i k \eta) e^{i k \eta}\right],
$$

where $\eta=-1 /(H a)$, and $\alpha_{\mathbf{k}}$ and $\beta_{\mathbf{k}}$ are constants which satisfy

$$
\left|\alpha_{\mathbf{k}}\right|^{2}-\left|\beta_{\mathbf{k}}\right|^{2}=1
$$

We shall choose $\left(\alpha_{\mathbf{k}}, \beta_{\mathbf{k}}\right)=(1,0)$ so that the vacuum reduces to the one in Minkowski spacetime at the short-wavelength limit $(-k \eta \rightarrow \infty)$. Therefore we obtain

$$
Y=\frac{i H}{\sqrt{2 k^{3}}}(1+i k \eta) e^{-i k \eta}
$$

\section{MATCHING THE SHORT- AND THE LONG-WAVELENGTH REGIMES: THE CASE OF A LINEAR POTENTIAL}

Having obtained the short- and the long-wavelength solutions we next connect these two regimes in order to determine the coefficients $c_{1}(k)$ and $c_{2}(k)$. In this section we consider the simplest case in which the inflaton's potential can be approximated by a linear function near the point where the inflaton switches its direction of motion. That is, we take

$$
V(\phi)=V_{0}+V^{\prime} \phi
$$

with $V_{0}$ and $V^{\prime}$ being constants and assume $\left|V^{\prime} \phi\right| \ll V_{0}$. Then the solution of Eq. (5) is

$$
\dot{\phi}(t)=\dot{\phi}_{s}\left(1-e^{-3 H\left(t-t_{0}\right)}\right)=\dot{\phi}_{s}\left[1-\left(\frac{a\left(t_{0}\right)}{a(t)}\right)^{3}\right],
$$

where $\dot{\phi}_{s} \equiv-V^{\prime} / 3 H$ is the velocity of $\phi$ in the slow-roll limit and we have set

$$
\dot{\phi}\left(t_{0}\right)=0 \text {. }
$$

In this case Eq. (17) is rewritten as

$$
Y(t)=c_{1}(k) \frac{\dot{\phi}_{s}}{H}\left[1-\left(\frac{a\left(t_{0}\right)}{a(t)}\right)^{3}\right]+c_{2}(k) \frac{-1}{3 \dot{\phi}_{s}}\left(\frac{k}{a(t)}\right)^{3},
$$

where we have chosen $T \rightarrow \infty$ so that $Y_{2}(t)$ becomes the pure decaying mode. It should be noted that it is nontrivial that $Y_{2}(t)$ is described like this all the time because the integrand is singular at $t=t_{0}[13]$. We emphasize that $Y_{1}(t)$, which is the nondecreasing mode, has a decaying component in contrast to the case of slow-roll inflation.

We next match Eq. (32) with the short-wavelength solution (28) via two different methods and confirm that both methods give the same results.

The first method we adopt is to use the series expansion of Eq. (28) with respect to $k / a(t)$, namely,

$$
Y=\frac{i H}{\sqrt{2 k^{3}}}\left[1+\frac{1}{2}\left(\frac{k}{a(t) H}\right)^{2}+\frac{i}{3}\left(\frac{k}{a(t) H}\right)^{3}+\cdots\right],
$$


and match each coefficient of the power series with that of the long-wavelength solution. As is seen here the zerothorder term matches the main part of the nondecreasing term and the third-order term corresponds to the decaying mode. However, the second-order term, which is present in Eq. (33), is absent in Eq. (32). In fact, this term should be regarded as a finite-wave-number correction to the nondecreasing mode $Y_{1}$ as we see now.

With the help of the $k=0$ solutions $Y_{1}(t)$ and $Y_{2}(t)$ and the Green's-function method, we obtain from Eq. (15) the following iterative expression for arbitrary $k[13]$ :

$$
\begin{aligned}
Y= & c_{1}(k) Y_{1}+c_{2}(k) k^{3} Y_{2}+k^{2} Y_{1} \int a Y_{2} Y d t-k^{2} Y_{2} \\
& \times \int a Y_{1} Y d t .
\end{aligned}
$$

The lowest-order iterative solution valid for small $k$ reads

$$
\begin{aligned}
Y(t) \cong & c_{1}(k) Y_{1}(t)+c_{2}(k) k^{3} Y_{2}(t)+c_{1}(k)\left(\frac{k}{a(t)}\right)^{2} \frac{\dot{\phi}_{s}}{2 H^{3}} \\
& +\mathcal{O}\left(\left(\frac{k}{a(t)}\right)^{5}\right)
\end{aligned}
$$

The new term corresponds to the second term in Eq. (33) and we can now determine $c_{1}(k)$ and $c_{2}(k)$ consistently up to the third order in $(k / a)^{3}$, to yield,

$$
\begin{gathered}
c_{1}(k)=\frac{i H}{\sqrt{2 k^{3}}} \frac{H}{\dot{\phi}_{s}}, \\
c_{2}(k)=\frac{\dot{\phi}_{s}}{\sqrt{2 k^{3}} H^{2}}\left[1-3 i\left(\frac{a\left(t_{0}\right) H}{k}\right)^{3}\right] .
\end{gathered}
$$

We note that $a\left(t_{0}\right) H / k=1$ for the mode leaving the Hubble radius when $\dot{\phi}$ vanishes.

Another method to decide $c_{1}(k)$ is simply to equate the value and the first time derivative of Eqs. (28) and (35) at the watershed. Because $M_{Y \text { eff }}^{2}$ is $\mathcal{O}\left(H^{2} / N\right)$, we can use the short-wavelength solution during $(k / a)^{2} \geq H^{2} / N$ and the long-wavelength solution for $(k / a)^{2} \lesssim H^{2} / N$. Thus we define the dividing epoch $\eta_{c}$ by $\left(k / a\left(\eta_{c}\right)\right)^{2}=H^{2} / N$ and match Eqs. (28) and (35) there.

At $\eta=\eta_{c}$ the short-wave solution (28) and its derivative read

$$
\begin{gathered}
Y\left(\eta_{c}\right)=\frac{i H}{\sqrt{2 k^{3}}}\left[1+\mathcal{O}\left(\left(-k \eta_{c}\right)^{2}\right)\right] \\
\frac{d Y}{d \eta}\left(\eta_{c}\right)-\frac{i H}{\sqrt{2 k^{3}}} k^{2} \eta_{c}\left[1-i k \eta_{c}+\mathcal{O}\left(\left(-k \eta_{c}\right)^{2}\right)\right]
\end{gathered}
$$

On the other hand, from Eq. (35) the long-wave counterparts are given by

$$
\begin{gathered}
Y\left(\eta_{c}\right)=c_{1}(k) \frac{\dot{\phi}_{s}}{H}\left[1+\frac{1}{2}\left(-k \eta_{c}\right)^{2}-\left(\frac{\eta}{\eta_{0}}\right)^{3}\right] \\
-c_{2}(k) \frac{H^{3}}{3 \dot{\phi}_{s}}\left(-k \eta_{c}\right)^{3}, \\
\frac{d Y}{d \eta}\left(\eta_{c}\right)=c_{1}(k) \frac{\dot{\phi}_{s}}{H}\left(k^{2} \eta_{c}-3 \frac{\eta_{c}^{2}}{\eta_{0}^{3}}\right)+c_{2}(k) \frac{H^{3}}{\dot{\phi}_{s}} k^{3} \eta_{c}^{2} .
\end{gathered}
$$

Equating Eq. (38) with Eqs. (40) and (39) with Eq. (41) we find

$$
c_{1}(k)=\frac{i H}{\sqrt{2 k^{3}}} \frac{H}{\dot{\phi}_{s}}\left[1+\mathcal{O}\left(\left(-k \eta_{c}\right)^{2}\right)\right] \text {, }
$$

$$
c_{2}(k)=\frac{\dot{\phi}_{s}}{\sqrt{2 k^{3}} H^{2}}\left[1-3 i\left(\frac{a\left(t_{0}\right) H}{k}\right)^{3}+\mathcal{O}\left(-k \eta_{c}\right)\right] \text {, }
$$

in agreement with Eqs. (36) and (37). Note that the leading terms of the above results are independent of the choice of the matching epoch $\eta_{c}$. Thus we find

$$
c_{1}(k)=-\frac{3 i H^{3}}{\sqrt{2 k^{3}} V^{\prime}} .
$$

\section{THE CASE OF A QUADRATIC POTENTIAL}

Next we consider the case for which the potential is locally given by

$$
V(\phi)=V_{0}-\frac{1}{2} m^{2} \phi^{2},
$$

near the turning point, where $V_{0}$ and $m$ are constants satisfying $H^{2} \equiv 8 \pi G V_{0} / 3 \gg m^{2}$. We assume $V_{0} \gg m^{2} \phi^{2}$. Then the solution of Eq. (5) reads

$$
\begin{gathered}
\dot{\phi}(t)=\frac{\lambda_{+}}{1-\frac{\lambda_{+}}{\lambda_{-}}} \phi_{0}\left(e^{\lambda_{+}\left(t-t_{0}\right)}-e^{\lambda-\left(t-t_{0}\right)}\right), \\
\lambda_{ \pm} \equiv-\frac{3}{2} H \pm \sqrt{\frac{9}{4} H^{2}+m^{2}},
\end{gathered}
$$

where we have set

$$
\begin{gathered}
\dot{\phi}\left(t_{0}\right)=0, \\
\phi\left(t_{0}\right)=\phi_{0} .
\end{gathered}
$$

By virtue of the inequality $H \gg m$ we find

$$
\lambda_{+} \cong \frac{m^{2}}{3 H^{2}}, \quad \lambda_{-} \cong-3 H .
$$


To the lowest order in $m^{2} /\left(3 H^{2}\right)$, Eq. (17) is expressed as

$$
Y=c_{1}(k) \frac{m^{2}}{3 H^{2}} \phi_{0}\left[1-\left(\frac{a\left(t_{0}\right)}{a(t)}\right)^{3}\right]-c_{2}(k) \frac{H}{m^{2} \phi_{0}}\left(\frac{k}{a(t)}\right)^{3} \text {. }
$$

We can repeat the same argument as the case of a linear potential. As is suggested by the fact that Eq. (51) coincides with Eq. (32) by the replacement

$$
-\frac{m^{2} \phi_{0}}{3 H}=-\frac{V^{\prime}\left[\phi_{0}\right]}{3 H} \Leftrightarrow \dot{\phi}_{s}=-\frac{V^{\prime}}{3 H},
$$

we obtain in this case

$$
c_{1}(k)=-\frac{3 i H^{3}}{\sqrt{2 k^{3}} V^{\prime}\left[\phi_{0}\right]} .
$$

Thus in both cases the coefficient of the nondecaying mode is given by the standard formula as if $\phi$ is slowly rolling with the speed

$$
\dot{\phi}=-\frac{V^{\prime}[\phi]}{3 H},
$$

even for the $k$ mode leaving the horizon when $\dot{\phi}$ vanishes.

\section{CONCLUSION}

In the present paper we have derived a formula for the spectrum of the density perturbation generated by inflation in the case where the time derivative of the inflaton $\dot{\phi}$ vanishes at some time during inflation. Calculation in such a situation is not straightforward because the amplitude of the growing mode in the long-wavelength limit vanishes at the turning point of the inflaton, which implies that the Bardeen parameter estimated for quantum fluctuations at the horizon crossing diverges at that time. This is the origin of the divergence of the standard formula at $\dot{\phi}=0$. Of course, the amplitude of perturbations generated around the turning point do not diverge in reality because the Bardeen parameter becomes finite when the inflaton moves away from the turning point. This implies that we must subtract a contribution of the decaying mode, for which the Bardeen parameter is not constant, from the perturbation generated from the quantum fluctuation in order to obtain a correct formula for the amplitude of the growing mode.

In order to treat this problem properly, we have first shown that the standard expression for quantum fluctuations in the short-wavelength limit gives a good approximation for the evolution of perturbations during inflation, at least in the case we are concerned with, even when wavelengths are much larger than the Hubble horizon size. Then we have matched this expression with an exact long-wavelength expression for the evolution of the perturbation variable, $Y$, which does not show any singular behavior when $\dot{\phi}$ vanishes, in a range of wavelength in which both expressions are valid to determine the amplitude of growing mode in quantum fluctuations. As a result we have obtained the new for- mula for the amplitude of the curvature perturbation

$$
\Phi\left(r=\frac{2 \pi}{k}\right)=\left.f \frac{3 H^{3}}{2 \pi\left|V^{\prime}[\phi]\right|}\right|_{t_{k}},
$$

where $f=3 / 5(2 / 3)$ in the matter- (radiation-) dominated stage. Apparently this formula coincides with the standard formula with $\dot{\phi}$ in the denominator replaced by the slow-roll velocity,

$$
\dot{\phi}=-\frac{V^{\prime}(\phi)}{3 H},
$$

but it is valid not only in the slow-roll regime but also in an extreme situation $\dot{\phi}=0$ when the relevant mode leaves Hubble radius during inflation.

Although we have done calculations only for two simple models in the present paper, the above formula seems to hold for perturbations generated around and after the turning point of the inflaton in generic models for the following reason. First recall that the expression (28) for perturbations generated from quantum fluctuations gives a good approximation during inflation even when the wavelength is much larger than the Hubble horizon size. Though it is a combination of a growing mode and a decaying mode at the horizon crossing, the amplitude of the latter component decreases rapidly with cosmic expansion after the horizon crossing. In that stage the Bardeen parameter $Z=-H Y / \dot{\phi}$ should approach a constant. As shown in the Appendix, in several Hubble time after passing the turning point, the slow-roll approximation becomes good and $\dot{\phi} / H$ is expressed as $-V^{\prime} / 3 H^{2}$, which is constant with a good accuracy in the early phase of inflation after the turning point. In the meanwhile $Y$ given by Eq. (28) also becomes a constant $i H / \sqrt{2 k^{3}}$. Hence, the value of the Bardeen parameter for the growing mode should be given by

$$
Z=-i \frac{H}{\sqrt{2 k^{3}}} \frac{3 H^{2}}{V^{\prime}} .
$$

This expression coincides with $Z$ obtained from $c_{1}(k) Y_{1}$ with $c_{1}(k)$ given by Eq. (36). This argument does not determine the amplitude of the decaying-mode component, but it has no importance in the estimation of the curvature perturbation in later states.

\section{ACKNOWLEDGMENTS}

J.Y. would like to thank the Isaac Newton Institute for Mathematical Sciences for its hospitality where this research was completed. This work was supported in part by the Monbusho Grant-in-Aid for Scientific Research Nos. 11740146 (J.Y.) and 11640273 (H.K.), and "Priority Area: Supersymmetry and Unified Theory of Elementary Particles (No. 707)" (J.Y.). 


\section{APPENDIX}

In this appendix, for inflation models in which $\dot{\phi}$ vanishes at some time during inflation and the inflaton potential $V$ satisfies condition (21), we show that $\left|\left(V^{\prime} /\left(\kappa^{2} V\right)\right)^{\prime}\right|$ is much smaller than unity around the turning point of the inflaton as well as in the slow-roll phase of the inflationary stage after that. We also show that a local quadratic approximation for the potential $V$ gives a good description of the evolution of the inflaton and the scale factor in the same period.

First we rewrite the basic evolution equations (4) and (5) in terms of the new variables

$$
\begin{gathered}
v=\kappa \frac{\dot{\phi}}{H}, \\
\zeta=\ln \left(a / a_{0}\right),
\end{gathered}
$$

as

$$
\begin{gathered}
\kappa \frac{d \phi}{d \zeta}=v, \\
\frac{d v}{d \zeta}=-3\left(1-\frac{v^{2}}{6}\right)(v+\mu),
\end{gathered}
$$

where $a_{0}$ is the value of $a$ at the time when $\dot{\phi}=0$. $H$ is written in terms of these variables as

$$
H^{2}=\frac{\kappa^{2} V}{3\left(1-\frac{v^{2}}{6}\right)} \text {. }
$$

Note that from the equation

$$
\frac{\dot{H}}{H^{2}}=-\frac{1}{2} v^{2}
$$

the inflation condition $(a H) \cdot>0$ is expressed as $v^{2}<2$.

For definiteness we consider the case $\mu>0$ at the turning point $\phi=\phi_{0}, v=0$. In this case $v$ becomes negative after the inflaton passes the turning point. We restrict the consideration to the inflationary stage $0 \leqslant \zeta \leqslant \zeta_{*}$, where $\zeta_{*}$ is the value of $\zeta$ at the time when $v$ becomes $-\sqrt{2}$ for the first time. In this stage Eq. (A4) yields

$$
\frac{d}{d \zeta}\left(1+\frac{v}{\mu}\right)=-3\left[1-\frac{v^{2}}{6}+\frac{\nu}{3}\left(1+\frac{|v|}{\mu}\right)\right]\left(1+\frac{v}{\mu}\right)+\nu
$$

where $\nu=-\mu^{\prime} / \kappa \geqslant 0$. From this equation we immediately see that $1+v / \mu$ cannot vanish. Hence Eq. (A4) is written as

$$
\frac{d|v|}{d \zeta}=3\left(1-\frac{v^{2}}{6}\right)(\mu-|v|) \text {. }
$$

From the condition $\nu^{\prime} \leqslant 0, \mu$ satisfies $\mu^{\prime} / \kappa \leqslant-\nu_{0}$, where $\nu_{0}$ is the value of $\nu$ at $\phi=\phi_{0}$. Integrating this equation, we obtain

$$
\mu \geqslant \mu_{0}+\nu_{0} \kappa\left(\phi_{0}-\phi\right)
$$

where $\mu_{0}=\mu\left(\phi_{0}\right)$. From this equation and Eq. (A8) it follows that $\phi$ satisfies the differential inequality

$$
\frac{d^{2} \phi}{d \zeta^{2}}+2 \frac{d \phi}{d \zeta}+\frac{2 \mu_{0}}{\kappa}+2 \nu_{0}\left(\phi_{0}-\phi\right) \equiv f \leqslant 0 .
$$

Solving this equation yields

$$
\begin{aligned}
|v(\zeta)|= & \frac{\mu_{0}}{\left(1+2 \nu_{0}\right)^{1 / 2}}\left(e^{\lambda_{1} \zeta}-e^{-\lambda_{2} \zeta}\right)-\frac{\lambda_{1}}{2\left(1+2 \nu_{0}\right)^{1 / 2}} \\
& \times e^{\lambda_{1} \zeta} \int_{0} d \zeta e^{-\lambda_{1} \zeta} f-\frac{\lambda_{2}}{2\left(1+2 \nu_{0}\right)^{1 / 2}} \\
& \times e^{-\lambda_{2} \zeta} \int_{0} d \zeta e^{\lambda_{2} \zeta} f
\end{aligned}
$$

where

$$
\lambda_{1}=\sqrt{1+2 \nu_{0}}-1, \lambda_{2}=\sqrt{1+2 \nu_{0}}+1 .
$$

Since $f \leqslant 0$, this equation gives the inequality

$$
|v| \geqslant \frac{\mu_{0}}{\left(1+2 \nu_{0}\right)^{1 / 2}}\left(e^{\lambda_{1} \zeta}-e^{-\lambda_{2} \zeta}\right) .
$$

Hence we obtain the estimate

$$
N \equiv \zeta_{*}<\frac{1}{2 \lambda_{1}} \ln \frac{2\left(1+2 \nu_{0}\right)}{\mu_{0}} \sim \frac{1}{\nu_{0}} .
$$

In particular it follows that $\nu_{0}$ should be much smaller than unity if a sufficient inflation occurs after the inflaton passes the turning point.

Next we show that $v / \mu$ rapidly approaches unity. Integrating Eq. (A7), we obtain

$$
1+\frac{v}{\mu}=e^{-3 g(\zeta)}+\int_{0}^{\zeta} d \zeta_{1} \nu\left(\zeta_{1}\right) e^{-3\left[g(\zeta)-g\left(\zeta_{1}\right)\right]},
$$

where

$$
g(\zeta)=\int_{0}^{\zeta} d \zeta_{1}\left[1-\frac{v^{2}}{6}+\frac{\nu}{3}\left(1+\frac{|v|}{\mu}\right)\right]
$$

Since $g,|v|$ and $\nu$ are nondecreasing functions of $\zeta$, this equation gives the inequality

$$
\begin{aligned}
1+\frac{v}{\mu} \leqslant & \frac{2 \nu}{6-v^{2}+2 \nu}+\frac{6-v^{2}}{6-v^{2}+2 \nu} \\
& \times \exp \left[-\frac{1}{2}\left(6-v^{2}+2 \nu_{0}\right) \zeta\right] .
\end{aligned}
$$

Therefore, we obtain

$$
1+\frac{v}{\mu} \lesssim \frac{\nu}{2},
$$




$$
\left|\frac{1}{v} \frac{d v}{d \zeta}\right| \lesssim \nu
$$

for $\zeta \gtrsim \zeta_{s} \equiv 1 / 3 \ln 3 / \nu$. Since $d v / d \zeta / v$ is expressed as

$$
\frac{1}{v} \frac{d v}{d \zeta}=\frac{\ddot{\phi}}{H \dot{\phi}}-\frac{\dot{H}}{H^{2}},
$$

the above estimate implies that the slow-roll approximation becomes good for $\zeta \geqslant \zeta_{s}$, i.e., in a few Hubble time after the inflaton passes the turning point.

Finally, we show that the linear approximation $\mu \simeq \mu_{0}$ $+\nu_{0}\left(\phi_{0}-\phi\right)$ for $\mu$ can be used to determine the evolution of the inflaton with a good accuracy for a long period after the inflaton enters the slow-roll regime. First, integrating Eq. (A3) by taking account of the inequality $|\nu|<\mu$, we obtain

$$
\frac{\nu}{\mu} \kappa\left|\phi-\phi_{0}\right| \lesssim \nu \zeta
$$

If we expand $\mu$ as

$$
\mu=\mu_{0}+\nu_{0} \kappa\left(\phi_{0}-\phi\right)+\cdots,
$$

the ratio of the second term and the subsequent higher-order terms is in general of the order of $\left(\nu_{0} / \mu_{0}\right) \kappa\left|\phi_{0}-\phi\right|$. Hence, the above estimate implies that the first two terms in this expansion gives a good approximation for $\mu$ in the period $\zeta<1 / \nu_{0}$, which covers a large fraction of the slow-roll stage.
[1] For a review of inflation, see, e.g., A. D. Linde, Particle Physics and Inflationary Cosmology (Harwood, Chur, Switzerland, 1990); K. A. Olive, Phys. Rep. 190, 181 (1990).

[2] S. W. Hawking, Phys. Lett. 115B, 295 (1982); A. A. Starobinsky, ibid. 117B, 175 (1982); A. H. Guth and S.-Y. Pi, Phys. Rev. Lett. 49, 1110 (1982).

[3] A. D. Linde, Phys. Lett. 108B, 389 (1982); A. Albrecht and P. J. Steinhardt, Phys. Rev. Lett. 48, 1220 (1982).

[4] A. D. Linde, Phys. Lett. 129B, 177 (1983).

[5] R. K. Sacks and A. M. Wolfe, Astrophys. J. 147, 73 (1967).

[6] C. L. Bennett et al., Astrophys. J. Lett. 464, L1 (1996).

[7] T. Damour and V. F. Mukhanov, Phys. Rev. Lett. 80, 3440 (1998).

[8] A. R. Liddle and A. Mazumdar, Phys. Rev. D 58, 083508
(1998); A. Taruya, ibid. 59, 103505 (1999).

[9] J. Yokoyama, Phys. Rev. D 58, 083510 (1998); 59, 107303 (1999).

[10] J. M. Bardeen, Phys. Rev. D 22, 1882 (1980).

[11] H. Kodama and M. Sasaki, Suppl. Prog. Theor. Phys. 78, 1 (1984).

[12] V. F. Mukhanov, Zh. Eksp. Teor. Fiz. 94, 1 (1988) [Sov. Phys. JETP 67, 1297 (1988)].

[13] H. Kodama and T. Hamazaki, Prog. Theor. Phys. 96, 949 (1996).

[14] Y. Nambu and A. Taruya, Prog. Theor. Phys. 97, 83 (1997).

[15] J. M. Bardeen, P. J. Steinhardt, and M. S. Turner, Phys. Rev. D 28, 679 (1983).

[16] J. Hwang, Phys. Rev. D 48, 3544 (1993). 